\title{
Models for cosmic ray interactions
}

\author{
S S OSTAPCHENKO \\ Forschungszentrum Karlsruhe, Institut für Kernphysik, 76021 Karlsruhe, Germany \\ D.V. Skobeltsyn Institute of Nuclear Physics, Moscow State University, \\ 119992 Moscow, Russia
}

Received XXX

\begin{abstract}
Contemporary models of hadronic interactions are reviewed. Basic phenomenological approaches are compared, with an emphasizes on the predicted air shower characteristics. Special attention is payed to the remaining discrepancies between present hadronic MC generators and cosmic ray data. Finally, future prospects concerning model improvements are discussed, in particular, regarding the possibilities to discriminate between different models on the basis of accelerator or cosmic ray measurements.
\end{abstract}

\section{Introduction}

Nowadays Monte Carlo (MC) models of hadronic interactions are extensively used in collider and cosmic ray (CR) physics, being applied for designing new experiments, analyzing and interpreting data, or for testing new theoretical ideas, when the latter are incorporated in the corresponding MC generators. Here the last option is more characteristic for accelerator physics. High energy cosmic rays are typically studied by means of extensive air shower (EAS) techniques: instead of detecting primary $\mathrm{CR}$ particles directly one measures different characteristics of the nuclear-electro-magnetic cascade, so-called air shower, induced by their interactions in the atmosphere. Because of a large number of hadronic collisions during such a cascade it is very difficult to disentangle partial contributions of interactions of particular energies and to test individual features of MC models. In CR physics hadronic generators are an important technical tool, which extrapolates current theoretical and experimental knowledge from the energies of present accelerators up to the highest $\mathrm{CR}$ energies and helps to establish a relation between the properties of primary cosmic ray particles and the observed EAS characteristics.

Are there significant differences between collider and cosmic ray models? Is it possible to apply popular accelerator codes for CR data analysis? As we shall discuss below, all contemporary hadronic MC generators are based on similar physics ideas. Both at colliders and in cosmic rays one employs various approaches to describe hadronic and nuclear interactions; in neither case there exist a preferred approach, inherent for this or that particular field. However, generally accelerator codes are not suitable for an immediate application in CR field, being unable to treat some necessary reactions, like interactions of pions and kaons with nuclei or minimum bias nucleus-nucleus collisions, to perform predictive calculations of total inelastic and diffractive cross sections, or to be extrapolated into the ultra-high energy domain. It is worth stressing again that cosmic rays experiments can test model validity only indirectly. Analyzing CR data one has to rely on model predictions, without 
real possibilities to re-tune model parameters or to refine corresponding algorithms.

The goal of this work is to review basic approaches employed in contemporary CR models, to analyze remaining problems and to outline promising directions for future model improvements and for model discrimination on the basis of accelerator and cosmic ray measurements.

\section{High energy interactions: "pure QCD" approach}

Nowadays perturbative quantum chromo-dynamics (pQCD) is the only strict theory which provides the basis for both qualitative and quantitative description of hadronic production in high energy reactions. However, precise pQCD calculations are only possible for a restricted class of processes, namely those which are characterized by a large momentum transfer $Q^{2}$, such that running coupling $\alpha_{s}\left(Q^{2}\right)$ becomes small and corresponding contributions can be re-summed to a reasonable accuracy [1. Thus, one can not apply it to general hadron-hadron processes, which are expected to be dominated by the "soft" (low $Q^{2}$ ) physics. On the other hand, in the very high energy limit one expects that the role of high virtuality (high $p_{t}^{2}$ ) processes greatly increases, as their contributions are enhanced both by large parton multiplicity and by large logarithmic ratios of transverse and longitudinal momenta for successive parton emissions 1] 2]. Hence, one may develop MC generators, which rely on the pQCD formalism in the description of "hard" (high $p_{t}$ ) processes while treating the "soft" ones in a simplified fashion, assuming that the latter play smaller and smaller role when the energy increases.

Quite often such generators are referred to as "pure QCD models". How much is defined there by the perturbative QCD? Typically the main pQCD input is the inclusive cross section for production of parton jet pairs with transverse momenta larger than some cutoff $Q_{0}$, given to leading logarithmic accuracy as

$$
\begin{aligned}
\sigma_{a d}^{\mathrm{jet}}\left(s, Q_{0}^{2}\right) & =\sum_{i, j=g, q, \bar{q}} \int \frac{d p_{t}^{2}}{p_{t}^{2}} \int \frac{d x_{i}^{+} d x_{j}^{-}}{x_{i}^{+} x_{j}^{-}} \frac{d \sigma_{i j}^{2 \rightarrow 2}\left(x_{i}^{+} x_{j}^{-} s, p_{t}^{2}\right)}{d p_{t}^{2}} \\
& \times f_{a}^{i}\left(x_{i}^{+}, p_{t}^{2}\right) f_{d}^{j}\left(x_{j}^{-}, p_{t}^{2}\right) \Theta\left(p_{t}^{2}-Q_{0}^{2}\right),
\end{aligned}
$$

where $s$ is the c.m. energy squared for the interaction, $d \sigma_{i j}^{2 \rightarrow 2} / d p_{t}^{2}$ is the lowest order parton-parton scattering cross section, and $f_{a}^{i}\left(x, q^{2}\right)$ is the parton $i$ ((anti-)quark or gluon) momentum distribution function (PDF) in hadron $a$, when probed at the virtuality scale $q^{2}$. Here partons $i$ and $j$ in Eq. (1), participating in the high $p_{t}$ jet production, originate from partonic cascades in hadrons $a$ and $d$, being emitted by parent partons of smaller $p_{t}$ and larger energy, those in turn being emitted by their own parents and so on. The whole process can be described as a parton ladder, which starts in hadrons $a$ and $d$ with partons of $p_{t}^{2} \simeq Q_{0}^{2}$.

Still, to develop a MC model one has to make a number of additional assumptions, which are the essence of the mini-jet approach 3. 4. employed in SIBYLL [5] 6] and in a number of popular collider models, like PYTHIA [] or HIJING [8]. First, as $\sigma_{a d}^{\text {jet }}\left(s, Q_{0}^{2}\right)$ increases with energy faster than the observed total cross sec- 


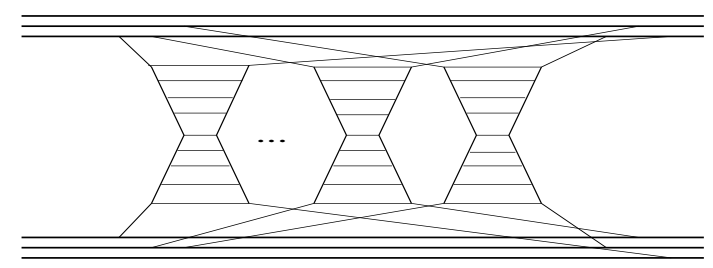

Fig. 1. Hadron-hadron scattering as multiple exchange of parton ladders.

tion, it appears mandatory to consider multiple jet production processes, in other words, to describe the interaction as multiple exchange of parton ladders, see Fig. 1]

Furthermore, one suggests that parton distribution in hadron $a$ is governed by its electro-magnetic form factor $\rho_{a}^{\mathrm{e} / \mathrm{m}}$ and, as a consequence, that at given impact parameter $b$ average number of jet production processes is defined by the product of $\sigma_{a d}^{\text {jet }}$ and the hadron-hadron overlap function $A_{a d}(b)=\int d^{2} b^{\prime} \rho_{a}^{\mathrm{e} / \mathrm{m}}\left(b^{\prime}\right) \rho_{d}^{\mathrm{e} / \mathrm{m}}\left(\left|\vec{b}-\overrightarrow{b^{\prime}}\right|\right)$ :

$$
\left\langle n_{a d}^{\text {jet }}\left(s, b, Q_{0}^{2}\right)\right\rangle=\sigma_{a d}^{\text {jet }}\left(s, Q_{0}^{2}\right) A_{a d}(b)
$$

Finally, assuming Poisson distribution for the number of jet processes at given $b$, with the average value $\left\langle n_{a d}^{\text {jet }}\left(s, b, Q_{0}^{2}\right)\right\rangle \equiv 2 \chi_{a d}^{\text {hard }}(s, b)$, and adding a similarly defined contribution of "soft" processes $\chi_{a d}^{\text {soft }}(s, b)=\frac{1}{2} \sigma^{\text {soft }}(s) A_{a d}(b)$, with a parameterized bare "soft" cross section $\sigma^{\text {soft }}(s)$, one can express total inelastic cross section via the eikonal $\chi_{a d}=\chi_{a d}^{\text {hard }}+\chi_{a d}^{\text {soft }}$ as 4,8 ,

$$
\sigma_{a d}^{\text {inel }}(s)=\int d^{2} b\left[1-e^{-2 \chi_{a d}(s, b)}\right]
$$

Using this scheme one can generate inelastic events, starting from sampling the impact parameter $b$ for the interaction, defining the number of hard processes, and generating parton cascades which preceed the hardest parton-parton scattering or which follow after it 7,8 . Then, one employs phenomenological procedures to convert final partons into hadrons, as well as to treat the soft part of the interaction. The most popular one is the string picture [9]: when final partons move apart, being connected to each other by color field, a color string is stretched between them. As long as the distance between partons increases so does the string tension, which finally gives rise to the break up of the string and to a multiple creation of quarkantiquark and diquark-antidiquark pairs, those forming secondary hadrons.

The disadvantage of the approach is a somewhat artificial separation of hard and soft processes and a rather arbitrary treatment for the latter. For the former one considers parton cascades as starting at given cutoff scale $Q_{0}^{2}$, thus neglecting the contribution of low $p_{t}\left(p_{t}<Q_{0}\right)$ partons.

\section{High energy interactions: Reggeon approach}

In Gribov's Reggeon approach [10 hadronic collisions are described as multiple scattering processes, where each elementary re-scattering corresponds to a micro- 
scopic parton cascade, just like in Fig. 1 Such an elementary process is treated there phenomenologically, as an exchange of a quasi-particle, the Pomeron, so that the scattering amplitude is the sum of contributions of graphs of Fig. 2

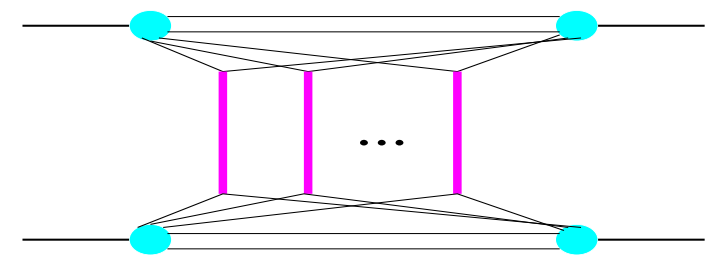

Fig. 2. Typical contribution to hadron-hadron scattering amplitude; elementary rescatterings are treated as exchanges of composite objects - Pomerons.

The Pomeron amplitude is postulated in the form [11]:

$$
\begin{aligned}
f_{a d}^{\mathrm{P}}(s, b) & =i \gamma_{a} \gamma_{d} s^{\alpha_{\mathrm{P}}(0)-1} / \lambda_{a d}(s) \exp \left[-b^{2} /\left(4 \lambda_{a d}(s)\right)\right] \equiv i \chi_{a d}^{\mathrm{P}}(s, b) \\
\lambda_{a d}(s) & =R_{a}^{2}+R_{d}^{2}+\alpha_{\mathrm{P}}^{\prime}(0) \ln s,
\end{aligned}
$$

which is characterized by a power-like energy rise and by a logarithmically increasing slope $\lambda_{a d}(s)$. Here $\alpha_{\mathrm{P}}(0), \alpha_{\mathrm{P}}^{\prime}(0)$ are the Pomeron trajectory parameters (intercept and slope) and $\gamma_{a}, R_{a}^{2}$ are the parameters for Pomeron-hadron $a$ coupling.

Having specified the structure of Pomeron-hadron vertices, one can obtain the total elastic amplitude for hadron-hadron scattering, hence, via the optical theorem, also total cross section. Applying the Abramovskii-Gribov-Kancheli (AGK) cutting rules [12, one can separate different contributions to the total cross section, corresponding to particular final channels of the reaction. For example, assuming eikonal vertices and neglecting diffraction dissociation and inelastic screening, one obtains Poisson distribution for the number of elementary production processes at given $b$ and arrives to Eq. (3) for inelastic cross section, with $\chi_{a d}(s, b)=\chi_{a d}^{\mathrm{P}}(s, b)$.

How to match this scheme with the pQCD treatment? In general, parton cascades contain both "hard" $\left(p_{t}^{2}>Q_{0}^{2}\right)$ and "soft" $\left(p_{t}^{2}<Q_{0}^{2}\right)$ parts, $Q_{0}$ being a chosen cutoff for pQCD being applicable. In the "semi-hard Pomeron" scheme, employed in QGSJET [13] and NEXUS [14] models, one applies phenomenological soft Pomeron treatment for the soft part of the parton cascade and describes general semi-hard processes as exchanges of a "semi-hard Pomeron", the latter being represented by a piece of QCD ladder sandwiched between two soft Pomerons [13, 15, 16, see Fig. 3] A general Pomeron appears to be the sum of the semi-hard and soft ones, the latter corresponding to a cascade of low $p_{t}\left(p_{t}<Q_{0}\right)$ partons.

The scheme coincides with the mini-jet approach in the treatment of the hard sector, represented by the ladder parts of the semi-hard Pomerons, see Fig. 3 . An important difference comes from considering multiple soft interactions, described as soft Pomeron exchanges, and from accounting for an additional source of particle production, which comes from the low $p_{t}$ part of the parton cascades ("soft preevolution") in semi-hard processes, shown as soft Pomeron "blobs" in Fig. 3] There 
Models for cosmic ray interactions

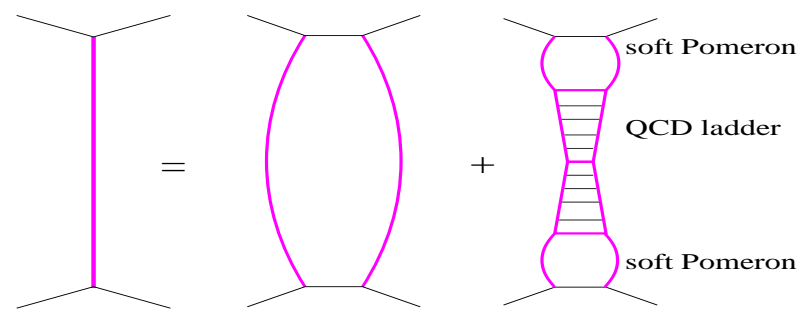

Fig. 3. A general "Pomeron" (1.h.s.) is the sum of the soft and the semi-hard ones correspondingly the 1 st and the 2 nd graph in the r.h.s.

is no artificial separation between soft and semi-hard processes, both being partial contributions to a general parton cascade, depending whether the latter develops in the low $p_{t}$ region or extends to higher virtualities, $p_{t}^{2}>Q_{0}^{2}$. The same soft Pomeron asymptotics is used to describe both soft processes and soft parts of semi-hard ones, defining in the latter case corresponding PDFs at scale $Q_{0}^{2}[14$, 16].

In general, there is no sharp border between the models of mini-jet or semihard Pomeron type. For example, modern version of SIBYLL [ 6 takes into account multiple soft interactions, described as Pomeron exchanges; DPMJET 17] considers an additional soft Pomeron exchange for each semi-hard process, which is not very different from the above-described "soft pre-evolution" treatment.

\section{Non-linear effects in high energy collisions}

Treating interactions at very high energies and small impact parameters one deals with the regime of high parton densities. There, individual parton cascades are no longer independent of each other; their overlap and mutual influence give rise to significant modifications of the picture described so far. At microscopic level such non-linear effects are described as merging of parton ladders [2], see Fig. [4
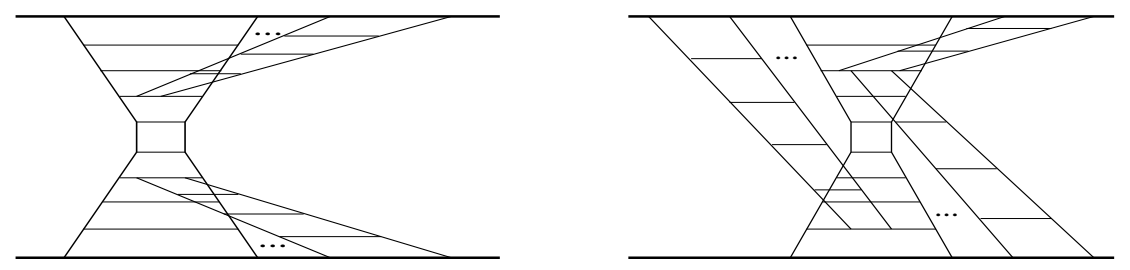

Fig. 4. Examples of diagrams which give rise to non-linear parton effects.

The corresponding pQCD treatment is based on the assumption that parton densities in the low virtuality region are saturated and any further parton branchings are compensated by the fusion of individual parton cascades [2. In mini-jet models one typically employs a phenomenological approach, introducing some parameterized energy-dependence of the $p_{t}$-cutoff for mini-jet production, 
$Q_{0}=Q_{0}(s)$, the latter being an effective saturation scale [6]. Still, completely neglecting the contribution of the saturated region (partons of $p_{t}<Q_{0}(s)$ ) to particle production may be a too strong assumption. On the other hand, applying the same $Q_{0}(s)$ cutoff to all collisions at a given energy appears to be a rather crude approximation; one loses correlations with actual parton densities, which depend on the parton Feynman $x$, on the impact parameters, and on the projectile and target mass numbers in case of nuclear collisions.

A dynamical description of non-linear interaction effects has been proposed in the QGSJET-II model [18, based on the assumption that saturation effects can be neglected for parton virtualities bigger than some fixed, energy-independent cutoff $Q_{0}$. In this scheme the multi-ladder graphs of Fig. 团 are replaced by enhanced diagrams, which correspond to Pomeron-Pomeron interactions [19, 20]. Suggesting that multi-Pomeron vertices are dominated by parton processes at comparatively low virtualities $\left|q^{2}\right|<Q_{0}^{2}$, one can re-sum all significant contributions of that kind and develop a self-consistent MC generation procedure for hadronic and nuclear collisions [18. The main parameter of the scheme, the triple-Pomeron coupling, has been inferred from HERA data on hard diffraction in deep inelastic scattering reactions. By construction, non-linear screening corrections appear to be correlated with corresponding parton densities and become larger at higher energies, smaller impact parameters, or for collisions of heavier nuclei, resulting finally in the saturation of PDFs at the scale $Q_{0}^{2}$ and in a considerable reduction of "soft" particle production. In particular, the predictions of the QGSJET-II model stay in agreement with the data of the RHIC collider on the multiplicity of secondary hadrons produced in central nucleus-nucleus interactions [21.

\section{Model sensitivity of air shower calculations}

Applying different models to air shower calculations one typically obtains some spread of the predicted EAS characteristics. To get insight into such differences, one compares different models with respect to the interaction characteristics which are most relevant for air shower development, i.e. regarding the predicted energy rise of inelastic cross sections, the relative energy loss of "leading" secondary particles (the inelasticity), or the multiplicity of produced secondaries. Such comparisons help to understand discrepancies between calculations and experimental data and to outline directions for future model improvements.

On the other hand, not every problem of EAS data interpretation may be attributed to potential model deficiencies. Nowadays there exist a bulk of accelerator data which seriously constrain predictions of hadronic models. Moreover, due to a large number of interactions in a hadronic cascade, observed air shower characteristics appear to depend rather on the general interaction trend over a wide energy range than on a sudden change of the interaction mechanism at a particular energy. In particular, comparatively low energy interactions, being well studied at accelerators, contribute with a large weight [22]. Thus, it would be naive to expect that some new valid model will change basic EAS predictions by a large amount. 
To quantify these statements one can perform simple tests with presently available models. For example, using the QGSJET model with the corresponding protonair cross section being artificially rescaled by $\pm 10 \%$, while keeping pion-air cross section unchanged, one obtains, due to the shift of the primary particle interaction point, about $10 \mathrm{~g} / \mathrm{cm}^{2}$ variation of the predicted shower maximum depth $X_{\max }$; the corresponding change of the charged particle number $N_{e}$ at ground ranges from $6 \%$ at $10^{16} \mathrm{eV}$ to $2 \%$ at $10^{19} \div 10^{20} \mathrm{eV}$, as shown in Fig. [5 For charged particle
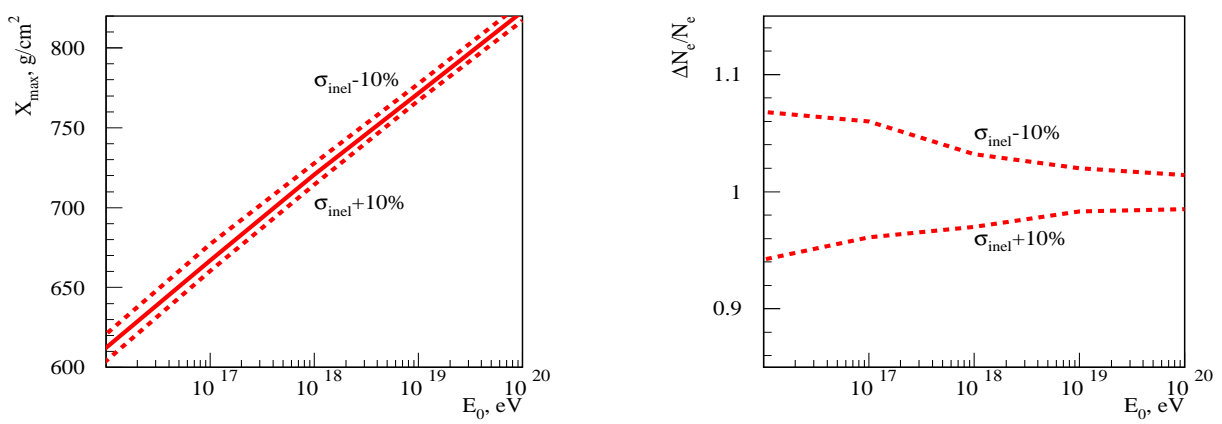

Fig. 5. Left: shower maximum depth for proton-induced EAS as calculated with the default QGSJET model (full line) and with $\pm 10 \%$ modifications of $\sigma_{\mathrm{p}-\text { air }}^{\text {inel }}$ (dashed lines). Right: variations of the calculated electron number at sea level for proton-induced vertical EAS for $\pm 10 \%$ modifications of $\sigma_{\mathrm{p}-\text { air }}^{\text {inel }}$ (relative to the default QGSJET).

density at $1000 \mathrm{~m}$ distance from the shower core $\rho_{\mathrm{ch}}(1000)$ such a sensitivity is even weaker, falling below $1 \%$ above $10^{18} \mathrm{eV}$, as the reduction (enhancement) of $N_{e}$ at ground is compensated by a flatter (steeper) lateral distribution of particles in case of larger (smaller) cross section. Thus, present experimental techniques, which reconstruct the primary energy on the basis of $\rho_{\mathrm{ch}}(1000)$, should not exhibit any significant model dependence. A similar conclusion holds for the fluorescence light-based energy reconstruction procedures [23].

Still, any studies of the cosmic ray composition, either based on $X_{\max }$ measurements or making use of electron-muon number correlations, exhibit significant model sensitivities. As for the shower muon number $N_{\mu}$ at ground level, it is very robust with respect to the discussed cross section variation, as shown in Fig. [6 (left), but depends on the multiplicity of hadronic interactions. One can consider two alternative modifications of model predictions - either increasing the multiplicity of just the primary particle interaction by a factor of two or enhancing the multiplicity of all secondary pion-air and kaon-air interactions by $10 \%$ - see Fig. 6 (right). As is easy to see from the Figure, both changes make a similar impact on the predicted EAS muon number at highest energies. Evidently, to obtain a factor of two increase of $N_{\mu}$ one needs a comparable enhancement of multiplicities of both primary and all secondary interactions, which would strongly contradict available accelerator data.

What are characteristic differences between presently available models? QGSJET and SYBILL predictions for $\sigma_{\mathrm{h} \text {-air }}^{\text {inel }}$ differ from each other by $10 \div 15 \%$ at $10^{19} \mathrm{eV}$; the disagreement for the multiplicity of charged particles $N_{\mathrm{p}-\text { air }}^{\mathrm{ch}}$ also increases with en- 

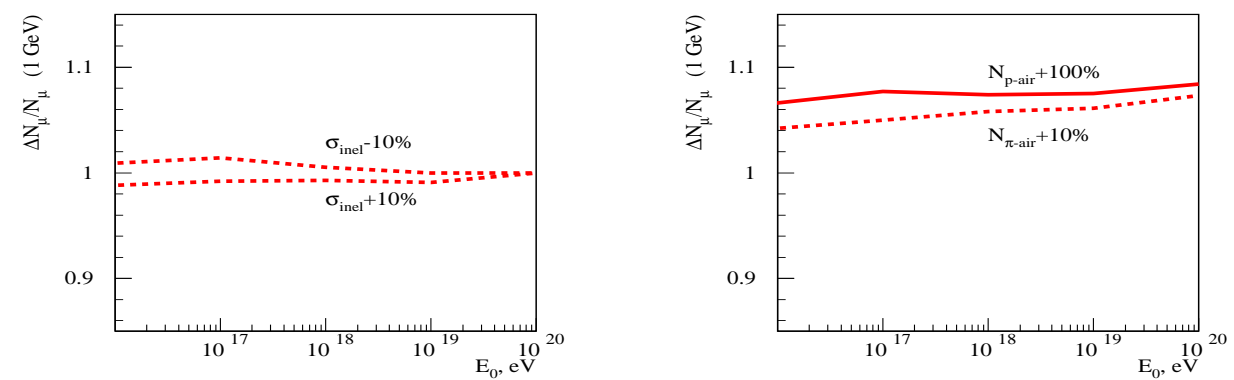

Fig. 6. Sensitivity of the muon number $\left(E_{\mu}>1 \mathrm{GeV}\right)$ at sea level for proton-induced vertical EAS to the cross section and multiplicity variations (as discussed in the text).
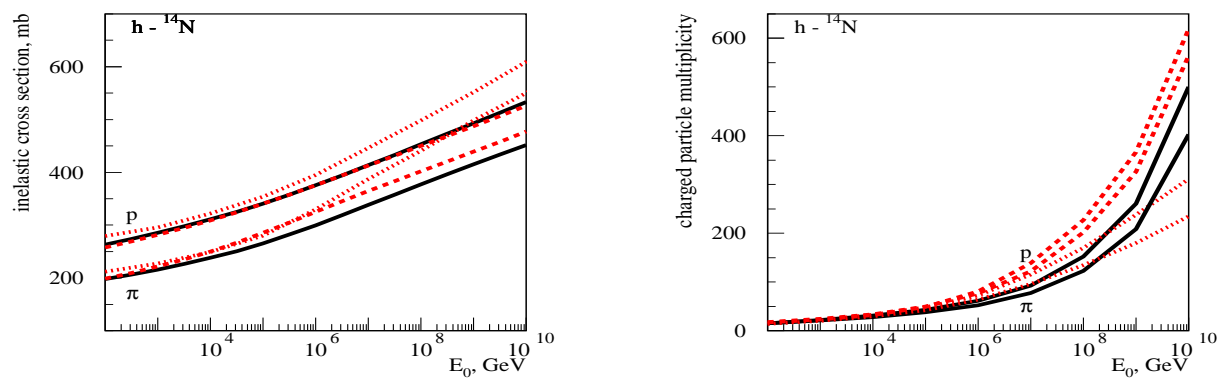

Fig. 7. Inelastic hadron-nitrogen cross section (left) and multiplicity of charged particles (right) for $p-{ }^{14} N$ and $\pi-{ }^{14} N$ collisions as calculated with the QGSJET-II, QGSJET, and SIBYLL models - full, dashed, and dotted curves correspondingly.

ergy, reaching a factor of two at ultra-high energies, as shown in Fig. 7 Main sources of these differences are the treatment of semi-hard processes in the models, which depends on the chosen PDFs, and the implementation of non-linear effects. The latter are not taken into account in QGSJET, which employs flat ("pre-HERA") parton distributions. SIBYLL uses realistically steep PDFs, which leads to a faster energy increase of $\sigma_{\mathrm{h} \text {-air }}^{\text {inel }}$. On the other hand, the faster multiplicity increase in QGSJET is due to the semi-hard Pomeron scheme employed, where additional particle production comes from the "soft pre-evolution", i.e. from hadronization of partons of small $p_{t}\left(p_{t}<Q_{0}\right)$. It is noteworthy that cross sections in QGSJETII are rather similar to the ones of QGSJET - see Fig. Z(left), despite the fact that the model uses realistic PDFs compatible with HERA data. This is because the effect of new PDFs is compensated by non-linear screening corrections. Such a compensation does not hold in SIBYLL, where such corrections are introduced via a $Q_{0}(s)$ cutoff for hard processes and are neglected for the "soft" component. On the other hand, non-linear effects reduce particle production in QGSJET-II compared to QGSJET, bringing it closer to SIBYLL - see Fig. 7(right). For EAS characteristics one obtains a rather large difference between QGSJET and SIBYLL predictions at highest energies, which reaches in particular $30 \%$ for the muon number. In turn, for QGSJET-II this difference is reduced to $10 \%$ [21]. 


\section{Some remaining problems}

Among the most remarkable recent results are KASCADE studies of electronmuon number correlations 24], which, apart from providing information on the spectra and mass composition of cosmic rays in the knee region, allowed one to quantify the discrepancies between present $\mathrm{MC}$ model predictions and $\mathrm{CR}$ data. The analysis showed that both QGSJET and SIBYLL have problems with the data interpretation. The corresponding discrepancies stay at the level of $10 \%$ for the predicted $N_{e}$ and $N_{\mu}$ and arise for the two models considered at different energy intervals. To reach a better agreement with the data it is desirable to have a model which predicts slightly deeper $X_{\max }$ compared to QGSJET, or, alternatively, reduces $N_{\mu}$, moving towards SIBYLL predictions. In fact, both holds in QGSJETII, where non-linear effects sizably reduce particle production compared to original QGSJET. Therefore, one may expect of it a somewhat better performance here.

Apart from that, present models seem to be unable to describe high multiplicity muon bundles observed by LEP collaborations [25]. One could think of improving the situation via a significant enhancement of multiplicity of hadronic interactions. On the other hand, as the highest multiplicity bundles mainly originate from events with the shower core inside the detector, one may expect a better performance from models which predict a deeper $X_{\max }$, hence, a steeper muon lateral distribution 25].

Finally, there exist a long-standing contradiction between ground-based and fluorescence-based EAS detection techniques, concerning both obtained CR spectra and mass composition [26. While the composition issue is still difficult to address, the energy reconstruction methods, as discussed above, should not exhibit significant model dependences. Hence, further studies of systematics of corresponding experiments seem to be desirable.

\section{$7 \quad$ Future prospects}

During last decade one observed a convergence of EAS predictions of contemporary cosmic ray interaction models. The remaining differences are still significant, resulting in particular in sizable uncertainties of $\mathrm{CR}$ composition studies. On the other hand, numerous new data, obtained with cosmic rays or at colliders, open the way for model discrimination and for their further development. Among the most important model uncertainties are those connected to the energy extrapolation of inelastic cross sections and of the inelasticity of hadron-air interactions. The new measurement of $\sigma_{\mathrm{h} \text {-air }}^{\text {inel }}$ by the HIRES collaboration [27] allows us to reduce the former and points towards smaller cross sections than those of present models. Future LHC measurements of $\sigma_{\mathrm{pp}}^{\text {tot }}$ will give us possibilities to calibrate reliably corresponding model procedures. On the other hand, RHIC collider provided valuable data on baryon "stopping" in central nucleus-nucleus collisions [28. When repeated for different centrality selections and for various combinations of projectile and target nuclei, those will allow us to fix reliably model predictions for the inelasticity of hadron-nucleus interactions.

On the theoretical side, of great importance is the pQCD treatment of non-linear 
interaction effects, in particular, concerning particle production in the transition region close to the parton saturation regime. Here, one may expect further progress to come from the "quasi-classical QCD" approach [29.

The author would like to acknowledge fruitful discussions with R. Engel.

\section{References}

[1] G. Altarelli: Phys. Rep. 81 (1982) 1.

[2] L.V. Gribov, E.M. Levin and M.G. Ryskin: Phys. Rep. 100 (1983) 1.

[3] L. Durand and P. Hong: Phys. Rev. Lett. 58 (1987) 303.

[4] T.K. Gaisser and T. Stanev: Phys. Lett. 219 (1989) 375.

[5] R.S. Fletcher et al.: Phys. Rev. D 50 (1994) 5710.

[6] R. Engel et al.: in Proc. 26th Int. Cosmic Ray Conf., Salt Lake City 1999, Vol. 1, p 415.

[7] T. Sjostrand and M. van Zijl: Phys. Rev. D 36 (1987) 2019.

[8] X.N. Wang: Phys. Rep. 280 (1997) 287.

[9] H. Bengtsson and T. Sjostrand: Comput. Phys. Commun. 46 (1987) 43.

[10] V.N. Gribov: Sov. Phys.-JETP 26 (1968) 414; 29 (1969) 483.

[11] A.B. Kaidalov and K.A. Ter-Martirosyan: Sov. J. Nucl. Phys. 39 (1984) 979.

[12] V.A. Abramovskii, V.N. Gribov and O.V. Kancheli: Sov. J. Nucl. Phys. 18 (1974) 308.

[13] N.N. Kalmykov, S.S. Ostapchenko and A.I. Pavlov: Bull. Russ. Acad. Sci. Phys. 58 (1994) 1966; Nucl. Phys. Proc. Suppl. B 52 (1997) 17.

[14] H.J. Drescher et al.: Phys. Rep. 350 (2001) 93.

[15] H.J. Drescher et al.: J. Phys. G 25 (1999) L91.

[16] S. Ostapchenko et al.: J. Phys. G 28 (2002) 2597.

[17] J. Ranft: Phys. Rev. D 51 (1995) 64.

[18] S. Ostapchenko: Nucl. Phys. Proc. Suppl. 151 (2006) 143; hep-ph/0501093

[19] O. Kancheli: JETP Lett. 18 (1973) 465

[20] A.B. Kaidalov, L.A. Ponomarev and K.A. Ter-Martirosyan: Sov. J. Nucl. Phys. 44 (1986) 468.

[21] S. Ostapchenko and D. Heck: in Proc. $28^{\text {th }}$ Int. Cosmic Ray Conf., Pune 2005.

[22] C. Meurer et al.: these proceedings; astro-ph/0512536

[23] T. Pierog et al.: these proceedings.

[24] T. Antoni et al. (KASCADE Collaboration): Astropart.Phys. 24 (2005) 1; H. Ulrich et al. (KASCADE Collaboration): these proceedings.

[25] P. Travnicek: these proceedings.

[26] A. Watson: Nucl. Phys. Proc. Suppl. 151 (2006) 83.

[27] K. Belov et al.: these proceedings.

[28] H. Yang: these proceedings.

[29] L.D. McLerran and R. Venugopalan: Phys. Rev. D 49 (1994) 2233. 